\title{
ARTICLE Genetic loss of GluN2B in D1-expressing cell types enhances long-term cocaine reward and potentiation of thalamo- accumbens synapses
}

\author{
Max E. Joffe ${ }^{1}$, Brandon D. Turner ${ }^{2}$, Eric Delpire ${ }^{3,4}$ and Brad A. Grueter (D) $^{1,2,3,4,5,6}$
}

Transient upregulation of GluN2B-containing NMDA receptors (R) in the nucleus accumbens (NAc) is proposed as an intermediate to long-term AMPAR plasticity associated with persistent cocaine-related behaviors. However, cell type- and input-specific contributions of GluN2B underlying lasting actions of cocaine remain to be elucidated. We utilized GluN2B cell type-specific knockouts and optogenetics to deconstruct the role of GluN2B in cocaine-induced NAc synaptic and behavioral plasticity. While reward learning was unaffected, loss of GluN2B in D1 dopamine receptor-expressing cells (D1) led to prolonged retention of reward memory. In control mice, prefrontal cortex (PFC)-D1(+) NAc AMPAR function was unaffected by cocaine exposure, while midline thalamus (mThal)-D1(+) NAc AMPAR function was potentiated but diminished after withdrawal. In D1-GluN2B ${ }^{-1-}$ mice, the potentiation of mThal-D1(+) NAc AMPAR function persisted following withdrawal, corresponding with continued expression of cocaine reward behavior. These data suggest NAc GluN2B-containing NMDARs serve a feedback role and may weaken rewardrelated memories.

Neuropsychopharmacology (2018) 43:2383-2389; https://doi.org/10.1038/s41386-018-0131-8

\section{INTRODUCTION}

Glutamatergic mechanisms of reward learning and processing contribute to the maladaptive behaviors implicated in neuropsychiatric disorders including addiction. While substances of abuse exert diverse effects throughout the nervous system, pathophysiological changes in nucleus accumbens (NAc) glutamate signaling are essential for many addiction-related behaviors [1-3]. Changes in $N$-methyl-D-aspartate receptor (NMDAR) and a-amino-3-hydroxy-5-methyl-4-isoxazolepropionic acid receptor (AMPAR) function temporally map onto different stages of drug experience [4-9]. Within days following cocaine experience, GluN2B-containing NMDARs are transiently upregulated in NAC medium spiny neurons (MSNs) $[4,10,11]$. Subsequently, a protracted drug-free period (withdrawal/abstinence) from cocaine induces a potentiation of synaptic AMPAR function in the NAC shell $[6,10,12-14]$ and core $[5,7,14,15]$ and is thought to be necessary for the incubation of drug craving and relapse-related behaviors [8, 16-20]. Transient cocaine-induced GluN2B upregulation in NAc MSNs is hypothesized to be necessary for subsequent potentiation of AMPAR and changes in conditioned drug-related behaviors [21-23].

MSNs provide the output of the NAc and can be readily (but not exclusively) dichotomized into two groups. D1-MSNs express D1 dopamine (DA) receptors (Drd1a, D1) and send projections to the ventral mesencephalon and the ventral pallidum [24], whereas D2MSNs express D2 DA and A2A adenosine (Adora2a, A2A) receptors and project to the ventral pallidum. While many nuanced findings have been reported, a simplified functional summary is that activation of D1-MSNs promotes reward-related behaviors, whereas activation of D2-MSNs attenuates reward and promotes anhedonia, anergia, and aversion $[25,26]$ Furthermore, AMPAR and NMDAR adaptations in response to cocaine exposure can be differentially expressed in NAc MSNs $[5,7,8,14,15,27]$. While pharmacological antagonism of NMDARs disrupts extinction of cocaine seeking [28], and NMDARs containing the GluN2B subunit are implicated in the formation of persistent cocaine-related memories $[4,16,18]$, the cell type-specific function of GluN2B in cocaine-mediated synaptic and behavioral adaptations is still unknown.

To investigate cell type-specific function of GluN2B, we crossed floxed Grin2b mice (GluN2B ${ }^{-1-}$ ) $[29,30]$ with mice expressing Cre driven by the D1 and A2A promoters [31]. Following viralmediated gene transfer of channelrhodopsin-2 (ChR2), we examined the effects of cocaine on reward learning and synaptic properties of these different cell populations and inputs in the NAc shell. We find that cocaine reward learning remained intact in both D1- and A2A-GluN2B ${ }^{-1-}$ mice. Surprisingly, while the cocaine-related memory dissipated in control (ctrl) mice following withdrawal, we find enhanced retention of cocaine conditioned place preference (CPP) in D1-GluN2B ${ }^{-1-}$ mice. We then examined cocaine-induced changes in AMPAR function in the NAc shell through input- and cell type-specific electrophysiology, focusing on inputs from the prefrontal cortex (PFC) or midline thalamus (mThal). At 2 weeks following non-contingent cocaine, AMPAR

\footnotetext{
${ }^{1}$ Department of Pharmacology, Vanderbilt University School of Medicine, Nashville, TN 37232, USA; ${ }^{2}$ Vanderbilt Brain Institute, Nashville, TN 37232, USA; ${ }^{3}$ Department of

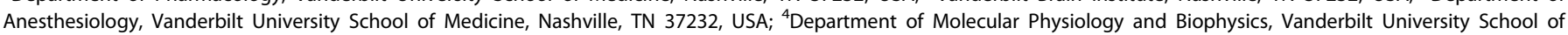

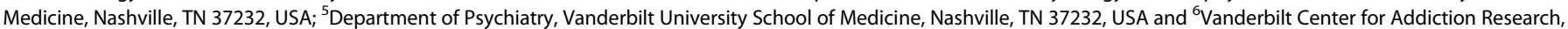
Vanderbilt University School of Medicine, Nashville, TN 37232, USA

Correspondence: Brad A. Grueter (brad.grueter@vanderbilt.edu)
}

Received: 7 March 2018 Revised: 16 May 2018 Accepted: 18 June 2018

Published online: 25 June 2018 
function was potentiated at mThal-D1(+) MSNs. The AMPAR potentiation dissipated at 4 weeks post cocaine, concurrent with the loss of cocaine reward memory. However, in D1-GluN2B ${ }^{-1-}$ mice, mThal-D1(+) NAc shell AMPAR function remained potentiated, consistent with the retention of cocaine reward memory. These results reveal a cell type-specific role of GluN2B in cocaineinduced functional adaptations of AMPARs and provide insight into the role of GluN2B in associative reward learning.

\section{METHODS}

Bacterial artificial chromosome (BAC) adult (6-12 weeks old) male mice on a C57BL/6J background were used in all experiments and were housed together in groups of two to five per cage on a 12/12 h light/dark cycle (lights on at 06:00), with food and water available ad libitum. Transgenic floxed mice (Grin2B $B^{\text {lox/lox }}$ ) were generated as previously described [30]. Grin2 $B^{\text {lox/lox }}$ mice were crossed with BAC transgenic mice expressing Cre recombinase under the regulation of Drd1a [32] or Adora2a promoter, analogous to that strategy we took with $\mathrm{Grin} 1^{f / f l}$ mice previously [33]. We elected to use Adora2a instead of the Drd2 promotor because D2 is expressed by striatal interneurons and midbrain dopaminergic neurons. Mice used for electrophysiology either expressed TdTomato under the control of the Drd1a promoter $[31,34]$ or expressed a Cre-inducible TdTomato (Ai9, The Jackson Laboratory, Sacramento, CA). Cre-negative littermates on the Grin $2 B^{\text {lox/lox }}$ background were used as controls. All experiments were conducted in a protocol approved by the Vanderbilt University institutional animal care and use committee.

Cocaine conditioned place preference

Prior to the place conditioning pretest, mice were habituated to behavioral testing with a $1 \mathrm{~h}$ exposure to similar activity chambers. All place conditioning sessions were $20 \mathrm{~min}$ in duration. Conditioning sessions were conducted twice daily, separated by $4 \mathrm{~h}$, and treatment order was counterbalanced across groups. All mice received injections of vehicle $(0.9 \%$ saline, $10 \mu \mathrm{L} / \mathrm{g}$, intraperitoneal) or cocaine $(20 \mathrm{mg} / \mathrm{kg})$ prior to confinement in one compartment. Overhead video recordings of mouse activity were analyzed with automated software (EthoVision XT, Noldus Information Technology, Leesburg, VA). The apparatus was divided into two contextually distinct environments based on floor and wall (rough with vertical bars vs smooth with checkerboard). No innate preference for either environment was observed and hence cocaine injections were paired with the less-preferred side for each mouse. We assessed submaximal cocaine CPP following one cocaine pairing and maximal cocaine CPP following three additional pairings. The acquisition of cocaine CPP was assessed the day after the last conditioning session and maintenance of the reward-related memory was assessed at extended time points.

\section{Stereotaxic injections}

At 4-5 weeks of age, mice underwent stereotaxic surgery for viralmediated gene transfer of ChR2. Bilateral $250-400 \mathrm{~nL}$ injections of AAV5-CaMKII-ChR2-EYFP (UNC Viral Vector Core, Chapel Hill, NC) were delivered into (relative to bregma): $m$ Thal (medial-lateral $(\mathrm{ML})$ : \pm 0.3 , anterior-posterior (AP): -1.2 , dorsal-ventral (DV): -3.0 ) or PFC (ML: $\pm 0.3, A P:-1.75$, DV: -2.75 ). Bilateral $800 \mathrm{~nL}$ injections of AAV5-CaMKII-Cre-GFP or AAV5-CaMKII-ChR2-EYFP were injected into the NAC (ML: \pm 0.75 , AP: 1.3 , DV: -4.5 ). Mice were killed for electrophysiology 4-6 weeks following surgery to allow for ChR2EYFP expression in axon terminals. Representative images of ChR2-EYFP injection sites can be found in a previous publication [35].

\section{Electrophysiology}

Detailed methodology for whole-cell voltage-clamp electrophysiology experiments can be found in a previous publication [5]. In brief, sagittal slices $(250 \mu \mathrm{m})$ were prepared containing the medial NAc shell. Only the first two slices $(<0.6 \mathrm{~mm}$ from midline) were used for recordings and the NAC shell was identified as being ventral and rostral to the anterior commissure. Recording pipettes were filled with a cesium-based internal solution (in $\mathrm{mM}$ ): 120 $\mathrm{CsMeSO}_{4}, 15 \mathrm{CsCl}, 8 \mathrm{NaCl}, 10$ HEPES, 0.2 EGTA, 10 TEA-Cl, 4 MgATP, $0.3 \mathrm{NaGTP}, 0.1$ spermine, and $5 \mathrm{QX}-314$. All recording solutions contained picrotoxin $(50 \mu \mathrm{M})$. NMDAR-excitatory postsynaptic potentials (EPSCS) were isolated in the presence of NBQX $(10 \mu \mathrm{M})$ and AMPAR-EPSCs in the presence of AP-5 $(50 \mu \mathrm{M})$. For current-voltage relationships, NMDAR-EPSCs and AMPAR-EPSCs were normalized to the peak amplitudes obtained at +30 and $-60 \mathrm{mV}$, respectively. For asynchronous EPSC (asEPSC) experiments, equimolar $\mathrm{Sr}^{2+}\left(2.5 \mathrm{mM} \mathrm{SrCl}_{2}\right)$ replaced $\mathrm{CaCl}_{2}$ in the extracellular solution and longer light durations were used (3-5 ms) to evoke glutamate release. Asynchronous events were identified using a predefined template search in the $200 \mathrm{~ms}$ window following EPSC onset [5,36]. The AMPAR rectification index was calculated as $\left(I_{+40}-I_{0}\right) /\left(I_{-40}-I_{0}\right)$. For cocaine experience experiments, mice received 5 injections of cocaine $(15 \mathrm{mg} / \mathrm{kg})$ in a locomotor activity apparatus.

\section{Data analysis}

Data are expressed as mean \pm SEM. For electrophysiology, each data point represents the average value from one cell. The number of cells and number of mice are represented as " $n$ " and " $N$ ", respectively. One- or two-way analysis of variance (ANOVA) or two-tailed Student's $t$-test were used when indicated. Repeated measures or paired tests were performed when appropriate. All post-tests employed Bonferroni corrections for multiple comparisons and $p<0.05$ was considered significant.

\section{RESULTS}

Verification of cell type-specific GluN2B deletions

To verify the specificity of the genetic deletions, we pharmacologically isolated NMDAR-EPSCs from NAc shell MSNs in acute brain slices identified by the fluorophore tdTomato (Fig. 1a). NMDAR-EPSCs from D1-GluN2B ${ }^{-1-}$ and A2A-GluN2B ${ }^{-1-}$ MSNs exhibited faster decay times than control (D1-GluN2B ${ }^{-1-}: 39.4 \pm$

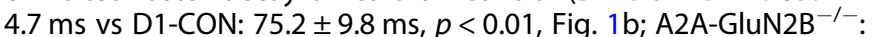
$31.2 \pm 5.9$ ms vs A2A-CON: $66.4 \pm 12.0 \mathrm{~ms}, p<0.05)$. D1-GluN2B ${ }^{-1-}$ and A2A-GluN2B ${ }^{-1-}$ MSNs also exhibited a blunted current-voltage relationship of NMDAR-EPSCs (Fig. 1b). These data are consistent with GluN2B-containing NMDARs exhibiting slower decay kinetics [37]. We also assessed the sensitivity of the NMDAR-EPSC to the GluN2B antagonist, ifenprodil. Ifenprodil reduced the NMDAR-EPSC in control D1 $(+)$ MSNs $(77.1 \pm 3.5 \%$ baseline). In contrast, ifenprodil did not affect the NMDAR-EPSC in MSNs with genetic deletion of GluN2B $(95.9 \pm 4.3 \%$ baseline, $p<$ 0.01 vs control), thus confirming cell type-specific GluN2B knockout.

\section{Enhanced retention of cocaine reward learning in D1-GluN2B ${ }^{-1-}$} mice

To determine consequences of cell type-specific GluN2B function on cocaine reward learning, we performed cocaine CPP in D1- and A2A-GluN2 $\mathrm{B}^{-1-}$ mice. A single-pairing protocol was used to assess submaximal reward learning. We observed a modest degree of cocaine CPP in control mice $(127 \pm 50 \mathrm{~s} / 10.5 \pm 4.2 \%$ session time, $p<0.05$, Fig. S1) with no difference across genotypes $(F(2,31)=$ 0.1282 , nonsignificant (n.s.)). We followed up with three additional cocaine/vehicle pairings to generate maximal CPP (303 $\pm 34 \mathrm{~s} / 25.2$ $\pm 2.8 \%$ session time, $p<0.05$, Fig. $2 \mathrm{a}$ ). All GluN2B ${ }^{-1-}$ genotypes exhibited CPP $(F(2,34)=0.4594$, n.s.), indicating that cell typespecific GluN2B function is not required for acquisition of cocaine CPP. 
A

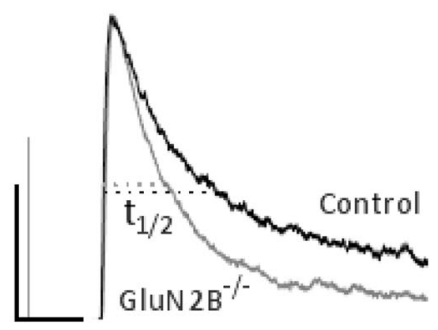

B

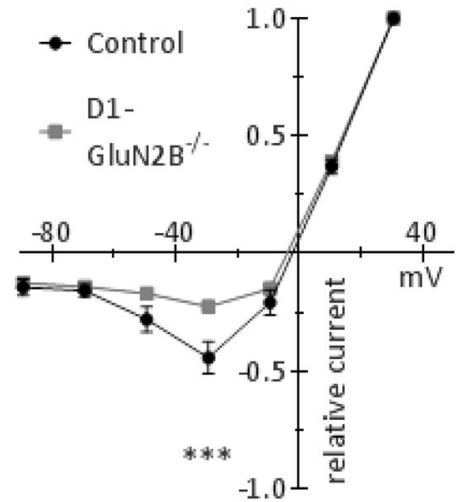

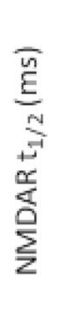

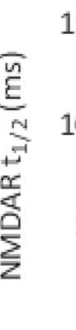

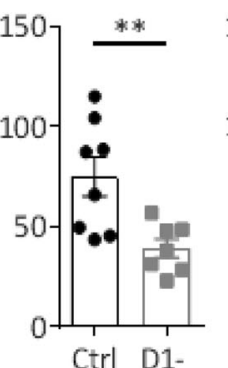

Ctrl D1-

GluN2B ${ }^{-/-}$
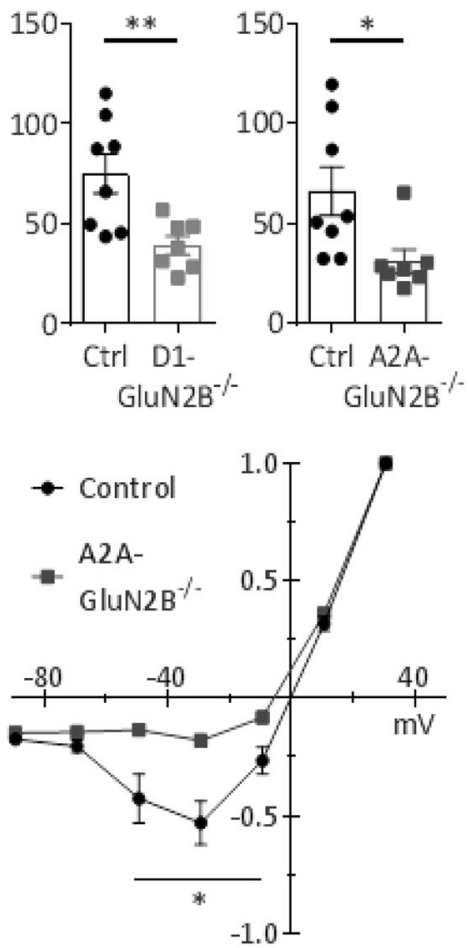

C Control

D1GluN2B $^{-/ *}$

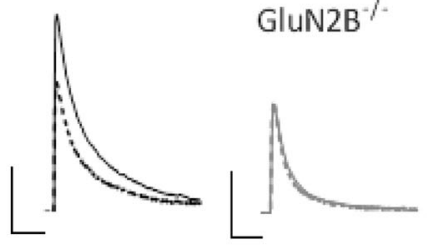

- Baseline - Baseline

-.. Ifenprodil -.. Ifenprodil

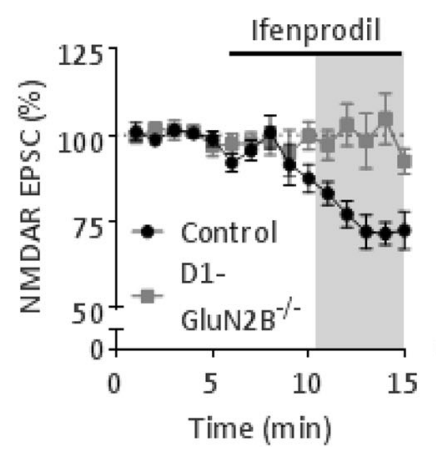

Fig. 1 Verification of cell type-specific GluN2B genetic deletions in the NAc. a Left, Representative pharmacologically isolated NMDAR-EPSCs recorded from fluorescently targeted D1(+) NAc MSNs. Scale bars denote $50 \mathrm{~ms}$ and $100 \mathrm{pA}$. Dotted lines denote time to $1 / 2 \mathrm{peak}\left(t_{1 / 2}\right)$. Right, Summary of the $t_{1 / 2}$ of the NMDAR-EPSC D1(+) MSNs (left; Ctrl, black circles, $n / N=8 / 3$; D1-GluN2B ${ }^{-\prime-}$, red squares, $\left.n / N=7 / 3\right)$ and D1 $(-)$ MSNs (right; Ctrl, black circles, $n / N=8 / 3$; A2A-GluN2B ${ }^{-1-}$, blue squares, $\left.n / N=7 / 3\right)\left({ }^{*} p<0.05,{ }^{* *} p<0.01, t\right.$-tests). b Summary of normalized, NMDAREPSC amplitudes as a function of membrane potential; D $1(+)\left(n / N=6 / 3, \mathrm{n} / N=7 / 3\right.$, left) and D $1(-)(n / N=8 / 3, n / N=6 / 3$, right $) \mathrm{MSNs}\left({ }^{*} p<\right.$ $0.05,{ }^{* *} p<0.001$, Bonferroni post-tests). c Left, Representative NMDAR-EPSCs inhibited by $10 \mu M$ ifenprodil. Middle, Timecourse displaying partial inhibition of NMDAR-EPSCs by ifenprodil in control D1(+) MSNs $(n / N=7 / 3)$ but not D1-GluN2B ${ }^{-1-}$ MSNs $(n / N=6 / 3)$. Right, Summary of last $5 \mathrm{~min}$ of recording. Deletion of GluN2B eliminates ifenprodil sensitivity in D1(+) MSNs (** $p<0.01, t$-test). Scale bars denote 100 ms and $100 \mathrm{pA}$

NAc GluN2B expression has been suggested to fulfill an intermediate state underlying the development of long-term behavioral effects of cocaine. Therefore, we sought to measure the maintenance of reward-related memory as assessed by retention of CPP 4 weeks later. We observed a main effect of genotype on the long-term maintenance of cocaine $\operatorname{CPP}(F(2,23)=8.186, p<$ 0.01 , Fig. $2 b$ ) with $D 1-G l u n 2 B^{-1-}$ mice exhibiting enhanced CPP at $4 \mathrm{r}$ weeks relative to controls and $A 2 \mathrm{~A}-$ Glun $2 \mathrm{~B}^{-1-}$ (D1: $384 \pm 59 \mathrm{~s}$ vs CON: $121 \pm 40 \mathrm{~s}, p<0.01$; A2A: $112 \pm 63 \mathrm{~s}, p<0.01)$. No difference was observed at a 2 -week time point $(F(2,17)=0.3959$, n.s., Fig. S2). To evaluate whether D1-Glun $2 \mathrm{~B}^{-\prime-}$ mice exhibited persistent memory retention, we compared the 4-week time point to the original test session revealing a significant interaction between time and genotype (Fig. $2 c$, d; $F(2,25)=9.778, p<0.001$ ). Specifically, at 4 weeks, control mice displayed a reduction in cocaine CPP (Maint: $121 \pm 40$ s vs Test: $303 \pm 34$ s, $p<0.01$ ), as did
A2A-GluN2B ${ }^{-1-}$ (Maint: $112 \pm 63$ s vs Test: $237 \pm 51$ s, $p<0.01$ ). In contrast, D1-GluN2B ${ }^{-1-}$ mice retained cocaine CPP at the 4-week time point (Maint: $384 \pm 59$ s vs Test: $296 \pm 40$ s, n.s.). Together, these data suggest that loss of GluN2B specifically from D1expressing cells enhances long-term retention of cocaine reward memory.

Intact locomotor sensitization in D1- and A2A-GluN2B ${ }^{-1-}$ mice In addition to measuring cocaine CPP, we also analyzed locomotor activity during conditioning sessions. During saline sessions, a two-way ANOVA revealed a main effect of genotype $(F(2,39)=$ $15.04, p<0.001$, Figure S2A) on locomotor activity. Post-tests indicated that $\mathrm{D} 1-\mathrm{GluN} 2 \mathrm{~B}^{-/-}$mice exhibited less locomotor activity and A2A-GluN2B ${ }^{-1-}$ mice displayed enhanced activity relative to control mice. Cocaine conditioning exhibited a main effect of session $(F(3,39)=39.64, p<0.0001$, Fig. S2B $)$ and 

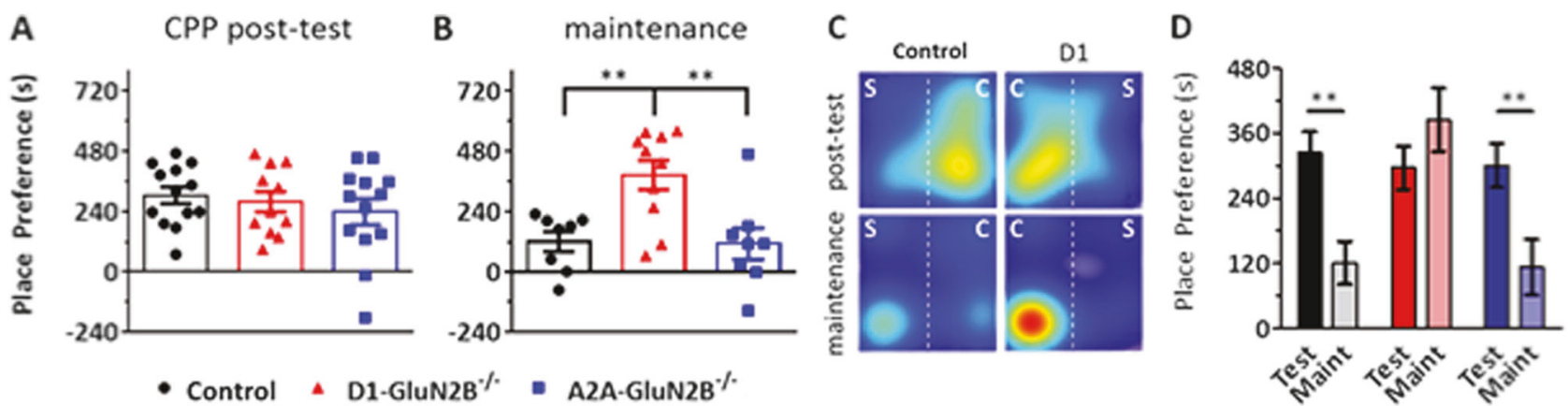

Fig. 2 D1-GluN2B ${ }^{-1-}$ display enhanced long-term retention of cocaine reward learning. a Cocaine place conditioning $24 \mathrm{~h}$ post cocaine pairing for $\mathrm{Ctrl}$ (black circles, $N=13$ ), D1-GluN2B ${ }^{-1-}$ (red triangles, $N=11$ ), and A2A-GluN2B ${ }^{-1-}$ (blue squares, $\left.N=13\right)$ mice. b Cocaine place conditioning at 4 weeks following cocaine exposure $\left(N=8,8,10,{ }^{* *} p<0.01\right.$, Bonferroni post-tests). c Representative heat maps displaying time spent on saline-paired (S) and cocaine-paired (C) sides during initial post-test (top) and 4-week maintenance test (bottom) in Ctrl (left) and D1GluN2B $^{-1-}$ (right) mouse. d Summary of initial post-test (dark) and maintenance test (light) CPP scores. Data were displayed in separate analyses in $(\mathbf{a}, \mathbf{b}){ }^{* *} p<0.01$, Bonferroni post-tests)

A
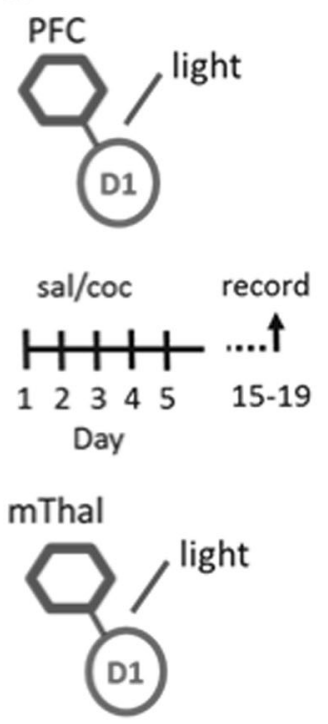

B

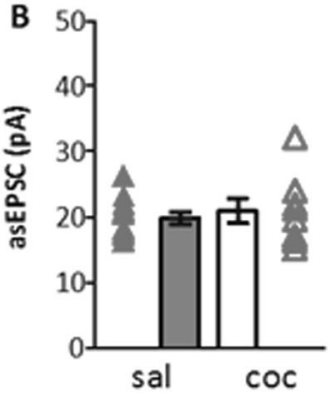

E

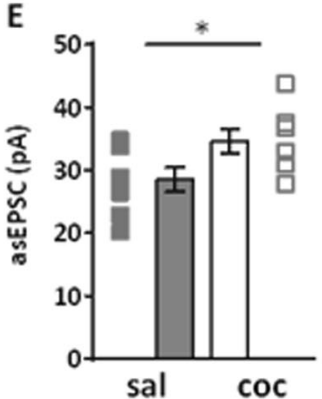

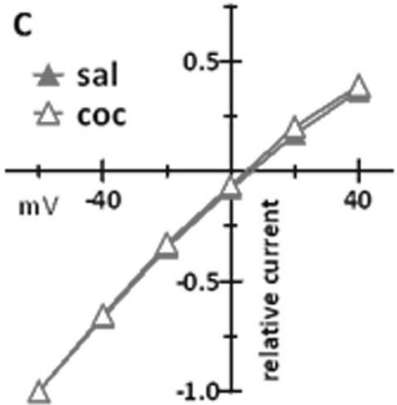

F

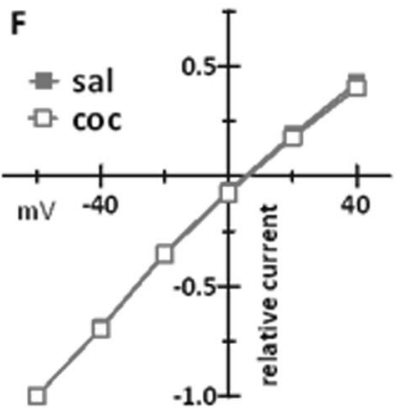

D

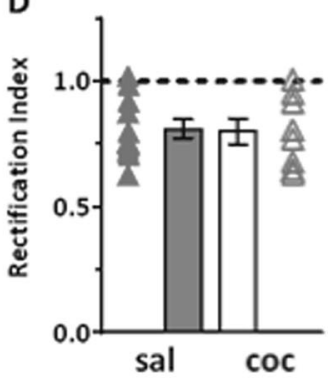

G

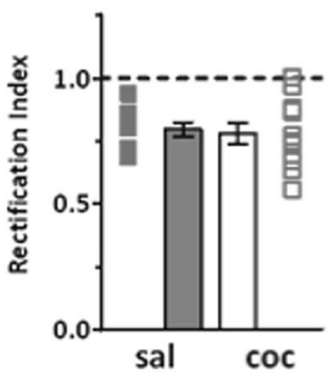

Fig. 3 Cocaine experience enhances AMPAR function at mThal-D1 NAc shell synapses without altering CP-AMPARs. a Temporal schematic of saline/cocaine injection and electrophysiological recordings from mice with viral-mediated expression of ChR2 in either the prefrontal cortex (PFC) (b-d) or midline thalamus (mThal) $(\mathbf{e}-\mathbf{g})$. b Amplitude of asynchronous EPSCs (asEPSCs) elicited in $\mathrm{Sr}^{2+}$-replaced aCSF in saline- (filled triangles) or cocaine-treated mice (open triangles) ( $n / N=11 / 4$ sal, $8 / 3 \mathrm{coc}$ ). c Pharmacologically isolated AMPAR current-voltage relationship normalized to the $-60 \mathrm{mV}$ peak current $(n / N=11 / 4 \mathrm{sal}, 8 / 3 \mathrm{coc})$. d Summary of AMPAR rectification index (RI) at PFC-D1( + ). e mThal-D1( + ) asEPSC amplitude of sal- (filled squares) or coc-treated mice (open squares ( ${ }^{*} p<0.05, n / N=8 / 4$ sal, $7 / 3$ coc). f AMPAR rectification at mThal-D1 synapses $(n / N=10 / 4 \mathrm{sal}, 11 / 4 \mathrm{coc}) . \mathbf{g}$ RI of mThal-D1(+) AMPAR-EPSCs

genotype $(F(2,39)=9.950, p<0.001)$ on locomotor activity. However, during the cocaine sessions, post-tests only revealed diminished hyperactivity in D1-GluN2 $\mathrm{B}^{-1-}$ mice. Importantly, despite displaying less locomotor activity than littermate controls, D1-GluN2B ${ }^{-1-}$ mice exhibited sensitization to the locomotor response (Coc 3: $82.4 \pm 18.0 \mathrm{~m}$ vs Coc 1: $28.0 \pm 6.8 \mathrm{~m}, p<0.01$ and Coc 4: $88.1 \pm 21.0 \mathrm{~m}, p<0.01$, Bonferroni post-tests). These data indicate that cell type-specific GluN2B function is not required for sensitization of locomotor activity, but does bidirectionally influence locomotion.

Hypoactivity in response to cocaine injections was recapitulated in a separate cohort of D1-GluN2B ${ }^{-I-}$ mice that underwent repeated cocaine injections in the same environment (genotype: $(F(1,15)=42.53, \quad p<0.0001$; interaction: $\quad(F(5,75)=4.971$, $p<0.001)$ ) (Figure S3A). We then aimed to test whether NAc- specific deletion of GluN2B is sufficient to attenuate the hyperactive locomotor response to cocaine. We virally expressed Cre recombinase in the NAc of 5-week old GluN2B ${ }^{\mathrm{f} / \mathrm{f}}$ mice and treated them with repeated cocaine injections 6 weeks later. NAcGluN2B ${ }^{-1-}$ mice displayed an attenuation of cocaine hyperactivity (genotype: $(F(1,18)=11.54, p<0.01$; interaction: $(F(5,90)=4.818$, $p<0.0001)$ ) (Figure S3B). Together, these data suggest that altered responses to cocaine in D1-GluN2B ${ }^{-1-}$ do not entirely stem from developmental changes, and that loss of GluN2B in the NAC is sufficient for these effects.

Cocaine-induced changes in NAc shell synaptic physiology mThal-D1(+) NAc shell AMPAR function is enhanced 2 weeks after cocaine exposure. Glutamatergic synaptic plasticity in the NAC has been demonstrated to mediate long-term conditioned 
A
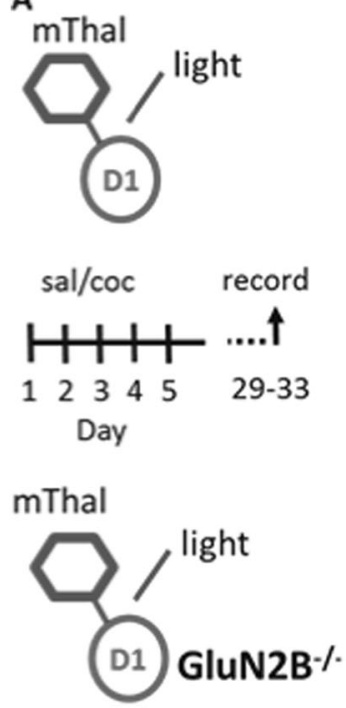

B

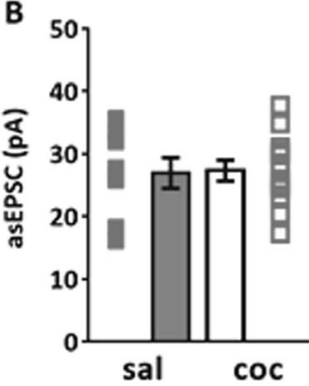

E

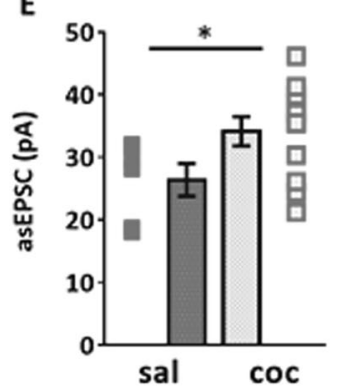

C

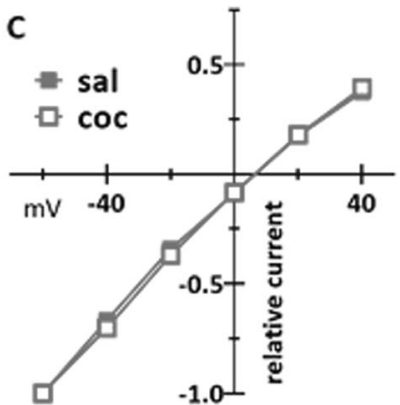

F

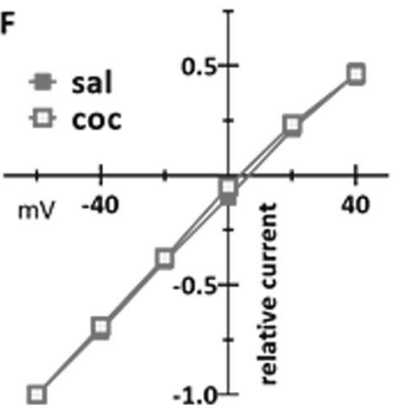

D

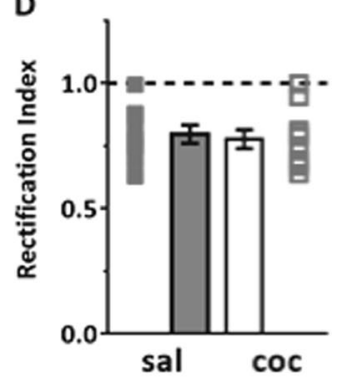

G

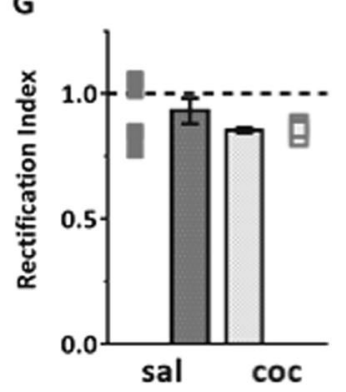

Fig. 4 D1-GluN2B ${ }^{-1-}$ mice retain mThal-D1 NAc shell AMPAR potentiation following long-term withdrawal from cocaine. a Temporal schematic of saline/cocaine injection and electrophysiological recordings from mice with viral-mediated expression of ChR2 in the midline thalamus (mThal) in control D1-TdTomato (b-d) or D1-GluN2B ${ }^{-1-}(\mathbf{e}-\mathbf{g})$ mice. b Amplitude of asynchronous EPSCs (asEPSCs) elicited in Sr ${ }^{2}$ ${ }^{+}$-replaced aCSF for saline- (filled squares) or cocaine-treated mice (open squares) ( $n / N=8 / 3$ sal, $12 / 4$ coc). c Isolated AMPAR current-voltage relationship normalized to the peak current obtained at $-60 \mathrm{mV}(\mathrm{n} / \mathrm{N}=9 / 3 \mathrm{sal}, 10 / 4 \mathrm{coc})$. d RI of AMPAR current-voltage relationships. e Summary of asEPSC amplitude in saline and cocaine D1-GluN2B ${ }^{-1-}$ mThal-D1 cells $\left({ }^{*} p<0.05, n / N=6 / 3\right.$ sal, $11 / 4$ coc). f Current-voltage relationship of AMPARs at mThal-D1 NAc shell synapses of GluN2B ${ }^{-1-}(n / N=6 / 3 \mathrm{sal}, 6 / 3 \mathrm{coc})$. $g$ Rl of AMPAR-EPSCs in GluN2B ${ }^{-1-}$

behavioral responses to cocaine $[8,16-18,20]$. Moreover, repeated cocaine exposure induced a transient increase in GluN2B expression and function in the NAc shell $[4,10,11]$. This transient increase is hypothesized to play a role in the development of cocaine locomotor sensitization and the incubation of cocaine craving - states that are both associated with upregulation of NAC shell AMPARs. Thus, we investigated the contribution of GluN2B to cocaine-induced adaptations in NAc shell AMPAR function in a cell type-specific manner at PFC and mThal inputs.

To model the cocaine exposure received during CPP, we conditioned mice with 5 days of cocaine in a novel environment and then 2 weeks of withdrawal (Fig. 3a). We recorded quantal-like asEPSCs from PFC and mThal inputs to NAc shell D1(+) MSNs using $\mathrm{Sr}^{2+}$-containing artificial cerebrospinal fluid (aCSF). The amplitude of asEPSCs reflects synaptic AMPAR function, and cocaine experience did not affect PFC-D1(+) asEPSC amplitude (Fig. 3b). No effect on AMPAR current-voltage relationship (Fig. 3c) or rectification index (Rl; Fig. 3d) was observed following noncontingent cocaine exposure. At 2 weeks after cocaine administration, the amplitude of mThal-D1 asEPSCs in the NAc shell was increased (coc: $34.6 \pm 2.0$ pA vs sal: $28.4 \pm 1.9$ pA, $p<0.05$, Fig. $3 e$ ) with no effect on current-voltage relationship (Fig. 3f) or Rl (Fig. 3g). Consistent with these findings, we did not observe cocaine-induced AMPAR rectification when eliciting EPSCs with non-specific electrical stimulation (Fig. S4). Moreover, we did not observe any effects of cocaine experience on mThal-D1(-) NAc shell AMPAR function (Fig. S3). The enhanced AMPAR-EPSCs at mThal-D1(+) MSNs are consistent with findings in non-cell-typespecific MSN recordings in the shell [10] and mThal-D1(+) MSNs in the NAc core [5].

D1-specific GluN2B KO prolongs cocaine-induced AMPAR plasticity. We determined that loss of GluN2B prolongs mThal-D1(+) AMPAR potentiation induced by cocaine exposure correlating with enhanced retention of CPP 4 weeks after cocaine exposure
(Fig. 4a). At this longer withdrawal time point, the asEPSC at mThal-D1(+) NAc shell synapses returned to saline control levels (coc: $27.3 \pm 1.6 \mathrm{pA}$ vs sal: $27.0 \pm 2.5 \mathrm{pA}$, n.s., Fig. $4 \mathrm{~b}$ ). Consistent with our data at the 2-week withdrawal time point, AMPAR current-voltage relationship and RI were not different following cocaine withdrawal (Fig. 4c, d). However, in the D1-GluN2B ${ }^{-1-}$ mice, the cocaine-induced potentiation of mThal-D1 AMPAR function persisted at 4 weeks of withdrawal (coc: $34.15 \pm 2.3 \mathrm{pA}$ vs sal: $26.38 \pm 2.5 \mathrm{pA}, p<0.05$, Fig. $4 \mathrm{e}$ ). RI of AMPAR-EPSCs in GluN2B $^{-1-}$ mice was not different than control (Fig. 4f, g). There was a trend towards increased sEPSC amplitude in cocaine-treated D1-GluN2B ${ }^{-1-}$ MSNs at four weeks (coc: $17.91 \pm 1.9 \mathrm{pA}$ vs sal: $12.56 \pm 1.4 \mathrm{pA}, \quad p<0.078$, Fig. S4B); otherwise the changes observed in D1-GluN2B ${ }^{-1-}$ mice do not reflect non-specific changes to D1-MSN AMPAR function (Fig. S5C-E). Taken together, these data indicate that increased mThal-D1(+) NAc shell AMPAR function is associated with the expression of cocaine CPP and that D1-specific loss of GluN2B enhances the persistence of cocaineconditioned synaptic function.

\section{DISCUSSION}

We demonstrate that, while the expression of cocaine CPP diminished over 4 weeks in controls, CPP remains intact in D1GluN2B ${ }^{-1-}$, mice suggesting that GluN2B serves as a break to limit or attenuate drug-induced adaptations. Additionally, GluN2B function in D1- and A2A-expressing cell types is not necessary for either cocaine reward learning or psychomotor sensitization. Upon assessment of the circuit and cell type-specific synaptic adaptations potentially underlying the cocaine-induced behaviors, no change to PFC-D1(+) NAc shell AMPAR function was observed following 2-week withdrawal from cocaine. However, mThal-D1(+) NAc shell AMPAR function was enhanced similar to findings in NAc shell and core $[5,10]$. AMPAR potentiation subsided at 4 weeks of withdrawal in control mice but persisted in 
D1-GluN2B ${ }^{-/-}$mice, suggesting mThal-D1(+) GluN2B regulation of AMPAR function in the NAC modulates retention of cocaine reward memory. It has been hypothesized that prolonged abstinence from cocaine exposure may induce a homeostatic scaling of AMPARs in the NAc [38] [23]. Furthermore, RNA interference knockdown of Grin2B leads to protein synthesisdependent increases in AMPAR homeostatic scaling in cortical neurons [38]. Thus, one possibility is that GluN2B negatively regulates homeostatic scaling of AMPARs induced by cocaine administration.

Consistent with reports from other brain regions [30, 40, 41], genetic loss of GluN2B function in NAc shell MSNs was associated with faster NMDAR decay kinetics. When assessing current-voltage relationship, we observed that loss of GluN2B attenuated the relative current passed at moderately hyperpolarized membrane potentials. Similar changes to the NMDAR current-voltage relationship in the NAc core have been associated with altered function of the GluN2C subunit following alcohol and cocaine exposure $[5,42,43]$. Together, these data suggest that triheteromeric GluN1/GluN2B/GluN2C NMDARs may be expressed in the NAC and underlie components of cocaine-induced plasticity. Future research investigating these NMDARs could elucidate the pathophysiology of addiction-related behavior and the identification of novel therapeutic targets.

At face value, the enhanced maintenance of cocaine reward learning exhibited by D1-GluN2B ${ }^{-1-}$ mice was reminiscent of the incubation of cocaine craving [44], a phenomenon that occurs following long-term abstinence from extended-access cocaine self-administration. Because this behavior is associated with enhanced NAC calcium-permeable (CP)-AMPAR expression $[12,16-18,20]$, we aimed to test the hypothesis that loss of GluN2B promoted incorporation of CP-AMPARs and related behaviors. We tested this by examining AMPAR rectification, as CP-AMPARs are known to display inward rectification at positive holding potentials. However, in agreement with previous work $[6,12,21,45]$ but see Mameli et al. 2009; Terrier et al. 2015), we found no evidence that non-contingent cocaine administration upregulated NAC shell CP-AMPAR function overall or at synapses from the PFC or mThal, even in the D1-GluN2B ${ }^{-/-}$. However, we did find further evidence that cocaine exposure potentiates mThal-D1(+) NAc function, expanding on previous work that examined mThal inputs to the NAc shell [10] and the NAc core [5].

We recently examined $\mathrm{D} 1-\mathrm{GluN1} 1^{-/-}$and $\mathrm{A} 2 \mathrm{~A}-\mathrm{GluN} 1^{-/-}$models and found that both mouse lines retained the expression of cocaine CPP [33]. The present data investigating GluN2B are consistent with those findings, which collectively indicate that development of cocaine CPP does not require intact NMDAR function in D1- or A2A-expressing cells. Utilizing a GluN2B antagonist, Ro-25,6981, infused into the NAc, Brown et al. [11] showed the development of cocaine-induced locomotor sensitization is dependent on GluN2B. However, it is less clear how these receptors contribute to synapse maturation in the stretch of time following withdrawal/abstinence in vivo. Beyond the drug intake period, GluN2B-containing NMDARs may actually serve to negatively regulate synaptic AMPAR expression $[23,38]$, potentially acting as a feedback mechanism to reduce drug-induced adaptations. Our results suggest that this may indeed be the case, as D1(+)-GluN2 $\mathrm{B}^{-/-}$mice retain cocaine-induced potentiation of AMPAR currents at mThal-D1(+) synapses and place preference to cocaine. It is worth noting that our results rely on genetically ablating GluN2B at the onset of D1 DA and A2A receptor expression which occurs early in development. Developmental adaptations may play a role in our results; however, no differences in AMPAR function were observed in naive or saline-treated GluN2B-deficient mice. It is also possible that D1-expressing cells outside of the NAc shell are contributing to the behavioral phenotype.
In summary, our results show that specific loss of GluN2B from D1-expressing cells enhances the retention of cocaine-induced conditioned behavioral and synaptic responses. Additionally, the data presented here suggest a correlative effect between AMPAR potentiation at mThal synapses onto NAc shell D1(+) MSNs and retention of cocaine CPP, although recent work from Shukla et al. [13] suggests cocaine CPP is not dependent on incorporation of CP-AMPARs. Together, these data raise the possibility that NMDARs containing GluN2B are expressed after highly salient experiences and weaken or even reverse those memories over time. Alternatively, a more durable reward memory could have been formed due to enhanced excitatory connectivity between mThal and D1(+) MSNs, consistent with the finding that GluN2B deletion in the adult PFC increased the strength of the thalamic input onto those neurons [46]. Furthermore, GluN2B knockdown increased the number of synaptic contacts in developing CA1 pyramidal neurons [47]. While the transient expression of GluN2Benriched silent synapses is thought to be essential in permitting long-term cocaine-induced adaptations [11, 16, 18, 22, 27], GluN2B may serve an additional feedback role simultaneously or at later time points, such as during extended abstinence. NAc NMDAR activity is considered to be a key player in the acquisition of reward-related memories and behaviors, but more experiments need to be performed to address whether NAc NMDARs may act to temporally restrict drug-associated reward and/or extinction learning. Input-specific investigation combined with cell typespecific deletions of NMDARs represent an attractive tool-set to carefully further elucidate the electrophysiological, biochemical, and morphological changes induced in NAC MSNs by rewarding experiences.

\section{ACKNOWLEDGEMENTS}

We thank Marina E Wolf and Yan Dong for helpful comments on the manuscript. This study was supported by the National Institute on Drug Abuse R00DA031699 (to BAG). The floxed Grin2b mouse was generated by the Gene-Targeted Mouse Core of the Integrative Neuroscience Initiative on Alcoholism (INIA)-stress consortium. The GeneTargeted Mouse Core is supported by NIH grant U01 AA013514 (to ED). Use of these mice requires a MTA from Eric Delpire (eric.delpire@vanderbilt.edu).

\section{AUTHOR CONTRIBUTIONS}

MEJ and BDT conducted the experiments; MEJ and BAG designed the experiments; ED provided the floxed Grin2b mouse; and MEJ, BDT, and BAG wrote the paper.

\section{ADDITIONAL INFORMATION}

Supplementary Information accompanies this paper at (https://doi.org/10.1038/ s41386-018-0131-8).

Competing interests: The authors declare no competing interest.

Publisher's note: Springer Nature remains neutral with regard to jurisdictional claims in published maps and institutional affiliations.

\section{REFERENCES}

1. Grueter BA, Rothwell PE, Malenka RC. Integrating synaptic plasticity and striatal circuit function in addiction. Curr Opin Neurobiol. 2012;22:545-51.

2. Joffe ME, Grueter CA, Grueter BA. Biological substrates of addiction. Wiley Interdiscip Rev Cogn Sci. 2014;5:151-71.

3. Turner BD, Kashima DT, Manz KM, Grueter CA, Grueter BA (2018). Synaptic plasticity in the nucleus accumbens: lessons learned from experience. ACS Chem Neurosci 2018. https://doi.org/10.1021/acschemneuro.7b00420. [Epub ahead of print].

4. Huang $Y H$, Lin $Y, M u P$, Lee BR, Brown TE, Wayman $G$, et al. In vivo cocaine experience generates silent synapses. Neuron. 2009;63:40-7.

5. Joffe ME, Grueter BA. Cocaine experience enhances thalamo-accumbens nmethyl-d-aspartate receptor function. Biol Psychiatry. 2016;80:671-81. 
6. Kourrich S, Rothwell PE, Klug JR, Thomas MJ. Cocaine experience controls bidirectional synaptic plasticity in the nucleus accumbens. J Neurosci. 2007;27:7921-8.

7. MacAskill AF, Cassel JM, Carter AG. Cocaine exposure reorganizes cell type- and input-specific connectivity in the nucleus accumbens. Nat Neurosci. 2014;17:1198-207.

8. Pascoli V, Terrier J, Espallergues J, Valjent E, O'Connor EC, Luscher C. Contrasting forms of cocaine-evoked plasticity control components of relapse. Nature. 2014;509:459-64

9. Thomas MJ, Beurrier C, Bonci A, Malenka RC. Long-term depression in the nucleus accumbens: a neural correlate of behavioral sensitization to cocaine. Nat Neurosci. 2001:4:1217-23

10. Neumann PA, Wang $Y$, Yan $Y$, Wang $Y$, Ishikawa $M$, Cui R, et al. Cocaine-induced synaptic alterations in thalamus to nucleus accumbens projection. Neuropsychopharmacology. 2016;41:2399-410.

11. Brown TE, Lee BR, Mu P, Ferguson D, Dietz D, Ohnishi YN, et al. A silent synapsebased mechanism for cocaine-induced locomotor sensitization. J Neurosci. 2011;31:8163-74.

12. McCutcheon JE, Wang $X$, Tseng KY, Wolf ME, Marinelli M. Calcium-permeable AMPA receptors are present in nucleus accumbens synapses after prolonged withdrawal from cocaine self-administration but not experimenter-administered cocaine. J Neurosci. 2011;31:5737-43.

13. Shukla A, Beroun A, Panopoulou M, Neumann PA, Grant SG, Olive MF, et al. Calcium-permeable AMPA receptors and silent synapses in cocaine-conditioned place preference. EMBO J. 2017;36:458-74.

14. Dobi A, Seabold GK, Christensen $\mathrm{CH}$, Bock R, Alvarez VA. Cocaine-induced plas ticity in the nucleus accumbens is cell specific and develops without prolonged withdrawal. J Neurosci. 2011;31:1895-904.

15. Bock R, Shin JH, Kaplan AR, Dobi A, Markey E, Kramer PF, et al. Strengthening the accumbal indirect pathway promotes resilience to compulsive cocaine use. Nat Neurosci. 2013;16:632-8.

16. Lee $B R$, Ma $Y Y$, Huang $Y H$, Wang $X$, Otaka $M$, Ishikawa $M$, et al. Maturation of silent synapses in amygdala-accumbens projection contributes to incubation of cocaine craving. Nat Neurosci. 2013;16:1644-51.

17. Loweth JA, Scheyer AF, Milovanovic M, LaCrosse AL, Flores-Barrera E, Werner CT, et al. Synaptic depression via mGluR1 positive allosteric modulation suppresses cue-induced cocaine craving. Nat Neurosci. 2014;17:73-80.

18. Ma YY, Lee BR, Wang X, Guo C, Liu L, Cui R, et al. Bidirectional modulation of incubation of cocaine craving by silent synapse-based remodeling of prefrontal cortex to accumbens projections. Neuron. 2014;83:1453-67.

19. Brebner K, Wong TP, Liu L, Liu Y, Campsall P, Gray S, et al. Nucleus accumbens long-term depression and the expression of behavioral sensitization. Science. 2005:310:1340-3.

20. Conrad KL, Tseng KY, Uejima JL, Reimers JM, Heng LJ, Shaham Y, et al. Formation of accumbens GluR2-lacking AMPA receptors mediates incubation of cocaine craving. Nature. 2008;454:118-21.

21. Boudreau AC, Wolf ME. Behavioral sensitization to cocaine is associated with increased AMPA receptor surface expression in the nucleus accumbens. J Neurosci. 2005;25:9144-51.

22. Lee BR, Dong Y. Cocaine-induced metaplasticity in the nucleus accumbens: silent synapse and beyond. Neuropharmacology. 2011;61:1060-9.

23. Wolf ME. Synaptic mechanisms underlying persistent cocaine craving. Nat Rev Neurosci. 2016;17:351-65.

24. Kupchik YM, Brown RM, Heinsbroek JA, Lobo MK, Schwartz DJ, Kalivas PW. Coding the direct/indirect pathways by D1 and D2 receptors is not valid for accumbens projections. Nat Neurosci. 2015;18:1230-2.

25. Lobo MK, Nestler EJ. The striatal balancing act in drug addiction: distinct roles of direct and indirect pathway medium spiny neurons. Front Neuroanat. 2011;5:41.

26. Smith RJ, Lobo MK, Spencer S, Kalivas PW. Cocaine-induced adaptations in D1 and D2 accumbens projection neurons (a dichotomy not necessarily synonymous with direct and indirect pathways). Curr Opin Neurobiol. 2013;23:546-52.

27. Graziane NM, Sun S, Wright WJ, Jang D, Liu Z, Huang YH, et al. Opposing mechanisms mediate morphine- and cocaine-induced generation of silent synapses. Nat Neurosci. 2016;19:915-25.
28. Hafenbreidel M, Rafa Todd C, Mueller D. Infralimbic GluN2A-containing NMDA receptors modulate reconsolidation of cocaine self-administration memory. Neuropsychopharmacology. 2017;42:1113-25.

29. Brigman JL, Daut RA, Wright T, Gunduz-Cinar O, Graybeal C, Davis MI, et al. GluN2B in corticostriatal circuits governs choice learning and choice shifting. Nat Neurosci. 2013;16:1101-10.

30. Brigman JL, Wright T, Talani G, Prasad-Mulcare S, Jinde S, Seabold GK, et al. Loss of GluN2B-containing NMDA receptors in CA1 hippocampus and cortex impairs long-term depression, reduces dendritic spine density, and disrupts learning. J Neurosci. 2010;30:4590-600.

31. Shuen JA, Chen M, Gloss B, Calakos N. Drd1a-tdTomato BAC transgenic mice for simultaneous visualization of medium spiny neurons in the direct and indirect pathways of the basal ganglia. J Neurosci. 2008;28:2681-5.

32. Gong S, Doughty M, Harbaugh CR, Cummins A, Hatten ME, Heintz N, et al. Targeting Cre recombinase to specific neuron populations with bacterial artificial chromosome constructs. J Neurosci. 2007;27:9817-23.

33. Joffe ME, Vitter SR, Grueter BA. GluN1 deletions in D1- and A2A-expressing cell types reveal distinct modes of behavioral regulation. Neuropharmacology. 2017;112:172-80.

34. Gong S, Zheng C, Doughty ML, Losos K, Didkovsky N, Schambra UB, et al. A gene expression atlas of the central nervous system based on bacterial artificial chromosomes. Nature. 2003;425:917-25.

35. Turner BD, Rook JM, Lindsley CW, Conn PJ, Grueter BA (2018b). mGlu1 and mGlu5 modulate distinct excitatory inputs to the nucleus accumbens shell. Neuropsychopharmacology 2018. https://doi.org/10.1038/s41386-018-0049-1. [Epub ahead of print].

36. Britt JP, Benaliouad F, McDevitt RA, Stuber GD, Wise RA, Bonci A. Synaptic and behavioral profile of multiple glutamatergic inputs to the nucleus accumbens. Neuron. 2012;76:790-803.

37. Paoletti $\mathrm{P}$, Bellone $\mathrm{C}$, Zhou Q. NMDA receptor subunit diversity: impact on receptor properties, synaptic plasticity and disease. Nat Rev Neurosci. 2013;14:383-400.

38. Sun $X$, Wolf ME. Nucleus accumbens neurons exhibit synaptic scaling that is occluded by repeated dopamine pre-exposure. Eur J Neurosci 2009;30:539-50.

39. Wang CC, Held RG, Chang SC, Yang L, Delpire E, Ghosh A, et al. A critical role for GluN2B-containing NMDA receptors in cortical development and function. Neuron. 2011;72:789-805.

40. Miller OH, Yang L, Wang CC, Hargroder EA, Zhang Y, Delpire E, et al. GluN2Bcontaining NMDA receptors regulate depression-like behavior and are critical for the rapid antidepressant actions of ketamine. Elife. 2014;3:e03581.

41. Wills TA, Klug JR, Silberman Y, Baucum AJ, Weitlauf C, Colbran RJ, et al. GluN2B subunit deletion reveals key role in acute and chronic ethanol sensitivity of glutamate synapses in bed nucleus of the stria terminalis. Proc Natl Acad Sci USA. 2012;109:E278-87.

42. Seif T, Chang SJ, Simms JA, Gibb SL, Dadgar J, Chen BT, et al. Cortical activation of accumbens hyperpolarization-active NMDARs mediates aversion-resistant alcohol intake. Nat Neurosci. 2013;16:1094-100.

43. Seif T, Simms JA, Lei K, Wegner S, Bonci A, Messing RO, et al. D-serine and Dcycloserine reduce compulsive alcohol intake in rats. Neuropsychopharmacology. 2015;40:2357-67.

44. Grimm JW, Hope BT, Wise RA, Shaham Y. Neuroadaptation. Incubation of cocaine craving after withdrawal. Nature 2001;412: 141-2.

45. Purgianto A, Scheyer AF, Loweth JA, Ford KA, Tseng KY, Wolf ME (2013). Different adaptations in AMPA receptor transmission in the nucleus accumbens after short vs long access cocaine self-administration regimens. Neuropsychopharmacology 2013;38:1789-97.

46. Miller $\mathrm{OH}$, Bruns $\mathrm{A}$, Ben Ammar I, Mueggler $\mathrm{T}$, Hall BJ. Synaptic regulation of a thalamocortical circuit controls depression-related behavior. Cell Rep. 2017;20:1867-80.

47. Gray JA, Shi Y, Usui H, During MJ, Sakimura K, Nicoll RA. Distinct modes of AMPA receptor suppression at developing synapses by GluN2A and GluN2B: single-cell NMDA receptor subunit deletion in vivo. Neuron. 2011;71:1085-101. 